\section{Reimar Banis}

\section{Den tieferen Ursachen auf der Spur}

Unbewusste Konflikte spielen nach den Theorien der Psychoanalyse eine bedeutende Rolle bei der Entstehung vieler körperlicher Krankheiten und seelischer Störungen. Da die betreffenden Konflikte im Unbewussten abgelagert und verdrängt sind, werden sie von der konventionellen Medizin in der Regel nicht erkannt und dementsprechend nicht behandelt. Auch bei Verhaltensstörungen, Erziehungsschwierigkeiten und vielen anderen psychologischen Problemen sollten unbewusste Konflikte als Ursache in Betracht gezogen werden. Das gilt insbesondere auch für «somatoforme» Störungen und schwer therapierbare Krankheitsbilder wie chronische Schmerzzustände oder Erschöpfungszustände ohne objektivierbare Ursache, bei denen eine Mitursache erfahrungsgemäss in solchen unbewussten Konflikten gefunden werden kann.

Konflikte wirken nach den Erfahrungen der Psychosomatischen Energetik (PSE) oft als Energieblockade,

\title{
Seelische Konflikte im Energiefeld erkennen und heilen
}

die dem Patienten viel Energie wegnimmt (Abb. 1). Energieblockaden wirken dabei als Heilhindernis, dessen Aufdeckung und Beseitigung häufig natürliche Heilprozesse wieder in Gang bringt. Oft verschwindet dadurch eine vorher bestehende Therapieresistenz, und viele Behandlungen wirken erst richtig. Dabei heilen nicht nur Krankheiten, sondern Menschen verändern sich in ihrer Gesamtheit zum Positiven, fühlen sich meist vitaler und empfinden oft mehr Lebensfreude. Sie sagen danach, dass sie ihr Leben deutlich besser in den Griff bekommen und ein besseres Selbstwertgefühl entwickelt haben, wobei solche Aussagen zeigen, dass ein solcher Therapieansatz ganzheitlich und vor allem auch psychotherapeutisch wirkt.

\section{Psychosomatische Energetik}

Bei der PSE handelt es sich um ein alternativmedizinisches Diagnose- und Therapiesystem, das ich vor ca. 15 Jahren entwickelt habe. Mit ihm kann man zuerst die Energielage eines

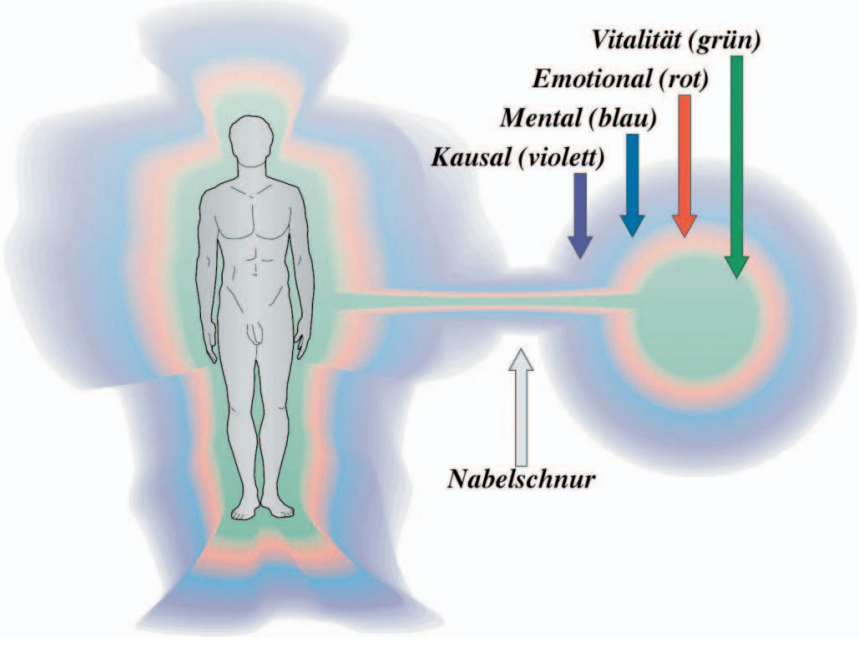

Abb. 1. Konflikt als Anhängsel der Aura, der mit einer Nabelschnur mit dem Konfliktträger verbunden ist und als Schmarotzer von dessen Energie lebt.
Menschen überprüfen, anschliessend seine seelischen Konflikte testen und diese danach mit bestimmten homöopathischen Komplexmitteln behandeln. Die Untersuchung mit dem Reba ${ }^{\circledR}$-Testgerät und speziellen Testampullen ist einfach und leicht $\mathrm{zu}$ erlernen. Der Therapeut testet mithilfe des Geräts die prozentuale Ladung der Lebensenergie. Dabei unterscheidet er die verschiedenen Schichten der feinstofflichen Aura: vital, emotional, mental und kausal. Jede der Energieebenen beinhaltet unterschiedliche stoffliche wie seelische Qualitäten. Das Testergebnis spiegelt erfahrungsgemäss das allgemeine Lebensgefühl und deckt sich ausserdem meist mit klinischen Diagnosen. So zeigen körperlich erschöpfte Menschen im PSE-Test meist eine niedrige vitale Ladung. Depressive Patienten haben meist einen reduzierten Emotionalwert, und bei Kranken mit einer Psychose findet man vergleichsweise geringe Werte im mentalen Bereich.

Durch die Testung der vier feinstofflichen Ebenen ermittelt der Therapeut in wenigen Minuten so etwas wie «Blutdruckwerte der feinstofflichen Ebenen». Die getesteten Werte erweisen sich als recht zuverlässig, wenn man die Testungen unterschiedlicher Untersucher vergleicht, und sie unterliegen kaum momentanen Schwankungen. Das Reba-Testgerät arbeitet mit präzise definierten Messsignalen, die mit den Hirnwellen in Resonanz gehen. Die Testergebnisse sind reproduzierbar, d.h., unterschiedliche Untersucher messen beim gleichen Patienten identische Werte. Um die Testung zu erlernen, sollte der Therapeut zuerst seine eigenen Kon-

\section{KARGER}

Fax +497614520714 Information@Karger.d www.karger.com (c) 2012 S. Karger GmbH, Freiburg www.karger.com/szg 
flikte behandelt haben und benötigt darüber hinaus eine ausführliche Schulung.

Im zweiten Schritt wird ermittelt, warum der Patient zu wenig Energie hat, was meist an den besagten Konflikten liegt. Um die Konflikte zu ermitteln, verwendet der Therapeut Ampullen mit homöopathischen Mitteln und prüft dabei zuerst die sieben Energiezentren (Chakren) auf Blockaden. Zeigt der Patient Reaktionen bei einer bestimmten Chakra-Ampulle, liegt im entsprechenden Energiezentrum eine Störung vor.

\section{Wie ein Konflikt entsteht}

Seelische Konflikte als Energieblockaden sind historisch seit Urzeiten bekannt. Schamanen der Urvölker sprechen davon, dass im Energiefeld des Kranken unsichtbare «Dämonen» und «Plagegeister» sitzen. Sie werden im schamanistischen Trancezustand als Giftschlangen, Spinnen und dergleichen visualisiert und sollen dem Kranken Lebenskraft rauben. Erst wenn der Medizinmann die Dämonen vertreibt, soll der Kranke genesen können. Heute sprechen wir nicht mehr von Dämonen, sondern von Konflikten. Konflikte entstehen durch traumatische Erlebnisse, die so bedrohlich wirken, dass sie seelisch kaum zu ertragen sind. Sie werden deshalb vom Gesamtorganismus kurzerhand ausgelagert (Abb. 1). Es handelt sich um das gleiche Phänomen, das von der Psychologie als «Verdrängung» bezeichnet wird. Um emotional zu überleben, verbannt man das Thema aus dem Bewusstsein. Daneben besitzt der Konflikt auch eine feinstoffliche Ladung, die auf der energetischen Ebene zu einer Auslagerung führt, was sich als Verlust von Lebensenergie auswirkt. Wie ein Vampir hängt der Konflikt anschliessend am Energiekörper des Patienten und zehrt von dessen Lebenskraft. Wenn sich daher jemand ständig müde und ausgelaugt fühlt, findet man meist Konflikte als eigentliche feinstoffliche Ursache. Logischerweise führt erst die Konfliktauflösung zur richtigen Erholung und zum Wiederauffüllen der Kraftreserven, d.h. nur so kann der Energieverlust ursächlich behandelt und dauerhaft beseitigt werden.

Die traumatischen Inhalte der Konflikte sind variantenreich, etwa Wut, Traurigkeit, Versagensängste und andere negative Gefühlsinhalte. Es gibt deshalb nicht einen Konflikt, sondern unterschiedliche traumatische «Gefühlspakete». Der Inhalt hängt überwiegend von der Situation $\mathrm{ab}$, in der der Konflikt entstanden ist. War man beispielsweise so wütend, dass der Organismus Gefahr lief, regelrecht zu explodieren, entstand ein Wutkonflikt. War man hilflos und glaubte, vernichtet $\mathrm{zu}$ werden, entstand ein Konflikt mit dem Thema «hilflos». Ich konnte 28 unterschiedliche Konfliktthemen unterscheiden. Sie können im Medikamententest (Abb. 2) mit bestimmten homöopathischen Komplexen, den sogenannten «Emotionalmitteln» Emvita ${ }^{\circledR}$, getestet werden. Konflikte halten dabei eine feststehende Rangfolge ein und sind den Chakren eindeutig zuzuordnen (Tab. 1). Sie sitzen am Energiesystem an ganz bestimmten Stellen, je nachdem um welchen Konfliktinhalt es sich handelt. Der Konflikt "Wut» findet sich beispielsweise immer im Bereich des Oberbauchs (3. Chakra), während Gefühlsinhalte von Hilflosigkeit im Beckenbereich (1. Chakra) angesiedelt sind.

\section{Bedeutung des Zentralkonflikts}

Da Konflikte eine feinstoffliche Natur besitzen, kann man ihre Grösse mit bestimmten Testsubstanzen messen. Ich habe spezielle Substanzen entdeckt, die den Konflikt kurzfristig vergrössern, so als legte man eine Lupe darüber. Hat der Test beispielsweise einen Konflikt mit dem Thema «Wut» aufgezeigt, bringt man die Testsubstanz Belladonna ins Energiefeld des Patienten. Nun lassen sich mit

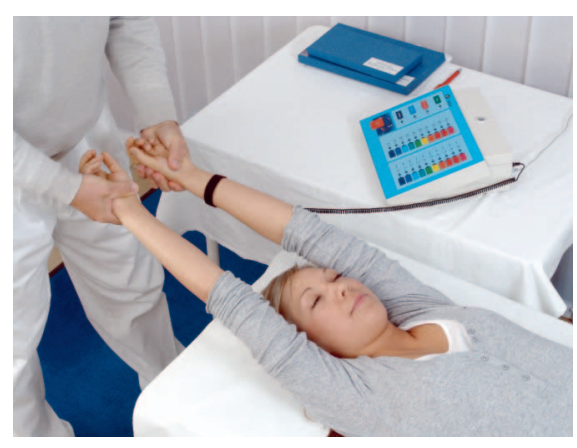

Abb. 2. Typische Testsituation bei der PSE mit kinesiologischem Armlängentest im Liegen.

dem Reba-Testgerät die vier Energieebenen der Wut ermitteln. So erfährt der Therapeut, wie viel vitale und emotionale Ladung der Konflikt hat, wie bewusst er ist (mentale Ladung) und wie hartnäckig seine Inhalte wiederholt werden (kausale Ladung). An der Konfliktgrösse kann man ablesen, welche Bedeutung der Konflikt für den Patienten hat. Der grösste aller vorhandenen Konflikte wird dadurch klar identifiziert. Er zeigt einen Kausalwert von über $80 \%$ bei sehr grossen Vital- und Emotionalwerten. Ich bezeichne ihn als "Zentralkonflikt», weil er eine zentrale Bedeutung für das Stoffwechselsystem des Patienten und auch für seinen Charakter hat. Das Chakra, dem der Zentralkonflikt zugeordnet wird, ist dabei entscheidend für das Temperament des Patienten: Sanguiniker (Hysteriker), Phlegmatiker (zwanghafter Typ), Choleriker (depressiver Typ) oder Melancholiker (schizoider Typ).

Den vier Typen entsprechen bestimmte Krankheitsdispositionen. Sanguiniker sind z.B. tendenziell aufbrausend und entweder «himmelhoch jauchzend» oder «zu Tode betrübt». Sie erleben alle Sinneseindrücke besonders intensiv und haben einen Hang zu Ausschweifungen. Für den Sanguiniker zählt überwiegend das Hier und Heute, und langfristiges Planen gehört eher nicht zu seinen Stärken. Der klassische Vertreter dieses Temperaments ist der charmante Lebemann, der alles in vollen Zügen geniesst und seine Sorgen mit «Wein, 
Weib und Gesang« betäubt. Von allen vier Charaktertypen findet man dabei variantenreiche Spielarten. Der erfahrene Therapeut erahnt das Temperament anhand eines bestimmten Grundthemas, das sich in verschiedenen Aspekten der Persönlichkeit und des Verhaltens zeigt.

\section{Typgerechte Beratung}

Mit der Typbestimmung erhält der Therapeut ein wertvolles Hilfsmittel zur tiefenpsychologischen Beratung, weil man durch die PSE-Testung viele unbewusste Wesenszüge der Persönlichkeit daraus ableiten kann, die man sonst nur nach intimer Kenntnis einer Person erhält. Dank der Kenntnis des Temperaments kann dem Patient eine typgerechte Lebensberatung angeboten werden. Jeder Charakter hat bestimmte «Laster», die gemieden werden sollten, und «Tugenden», die er fördern sollte. Am besten gelingt eine seelische Reifung durch die Abschmelzung des Zentralkonflikts, aber der Patient muss selbstverständlich auch selbst etwas dazu beitragen, damit es seelische Fortschritte gibt. Beim Testen des Charakters von Kindern kann man Eltern präzise Erziehungsratschläge geben, die dem Charaktertyp ihres Kindes entsprechen. Bei Beziehungsproblemen von Paaren erweisen sich Empfehlungen oft als hilfreich, wie man mit dem Charaktertyp des Partners am besten zurechtkommt, da bestimmte Charaktere erfahrungsgemäss besser oder schlechter miteinander auskommen und jeder Typ daher ein bestimmtes Vorgehen braucht.

\section{Klare Aussagen zu Fortschritt und Dauer der Therapie}

Durch das Messen der Konfliktgrösse wird der Therapiefortschritt nachvollziehbar dokumentiert, was dem Anwender eine verlässliche Aussage zur voraussichtlichen Therapiedauer erlaubt. Das erweist sich für den Therapeuten wie für den Patient gleicher-

Tab. 1.

Die 7 Chakren und ihre zugehörigen Konflikte

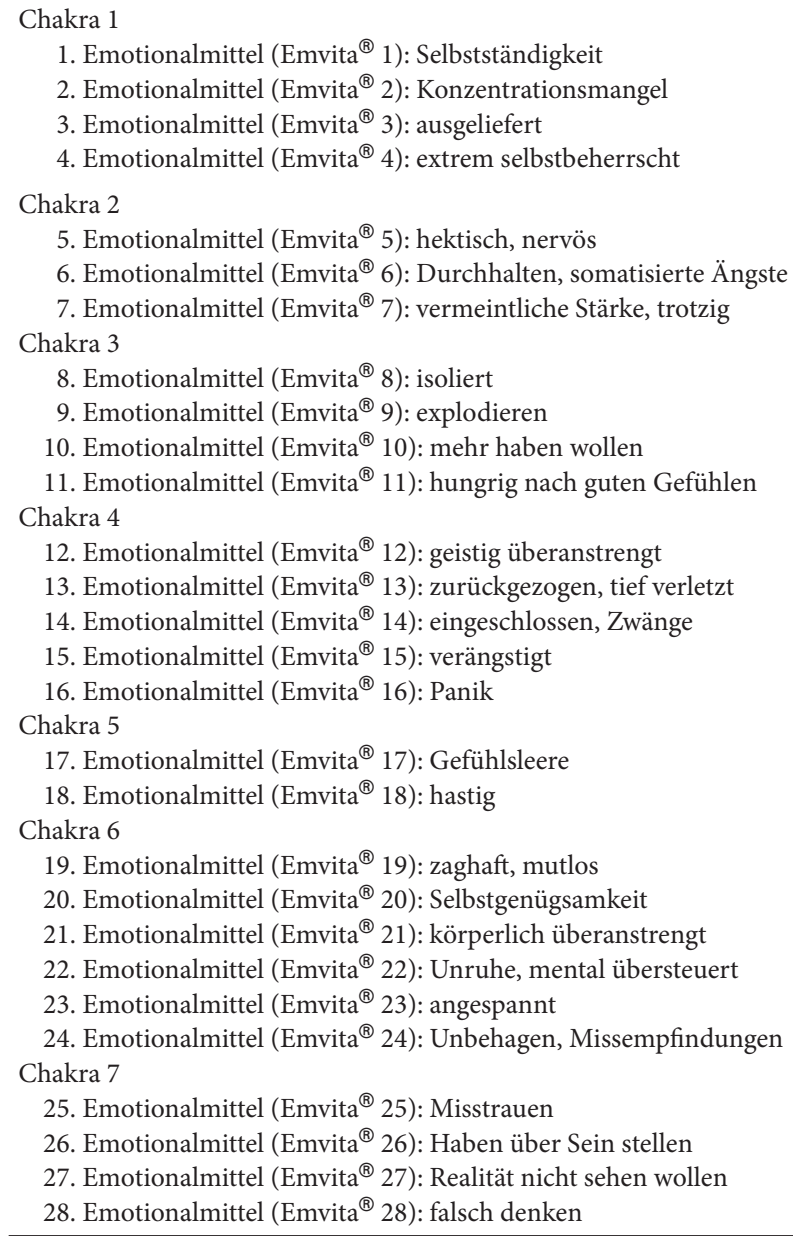

massen als hilfreich, da man die optimale Therapiedauer abschätzen kann. Am Ende der Testung überprüft man im sogenannten «Medikamententest», welche individuellen Heilmittel der Patient zusätzlich noch benötigt, und kann testen, wie stark er davon profitieren wird. Dazu legt man dem Patienten die Heilmittel in sein Energiefeld und misst mit dem Reba-Testgerät, wie stark sich die Energiewerte verändern. Meist benötigt er das betreffende Emotionalmittel (Emvita) und das Chakra-Medikament $\left(\right.$ Chavita $\left.^{\odot}\right)$, das zu seinem Konflikt gehört. Mit dem Reba-Testgerät ist es grundsätzlich möglich, sämtliche Heilmittel und Therapieverfahren auf ihre energetische Wirksamkeit prüfen. Man kann also auf einfache Weise vorhersagen, wie stark dem Patienten welche Behandlung helfen wird - oder auch, welche Substanzen ihm eher schaden bzw. Nebenwirkungen hervorrufen.

\section{Das Auflösen der Konflikte}

In der PSE wird der Konflikt energetisch aufgelöst. Der Patient nimmt über einige Wochen oder Monate homöopathische Komplexmittel: die Emotional- und Chakramittel Emvita und Chavita. Sie gehen mit dem Konflikt in Resonanz und lösen ihn nach und nach auf. Im Gegensatz zur Psychoanalyse braucht der Patient sich mit den belastenden Themen nicht bewusst auseinanderzusetzen. Selbst bei ganz kleinen Kindern und Tieren erlebt man gute Heilresultate, wenn übliche psychotherapeutische Techniken nicht angewandt werden können. Im Zuge des Heilprozesses kommt es häufig zu heftigen Träumen und gelegentlich anfänglich zu kurzfristigen seelischen Turbulenzen, etwa in Form einer kurzfristigen verstärkten Reizbarkeit. Bei dem Gros der Patienten verläuft der Heilprozess 


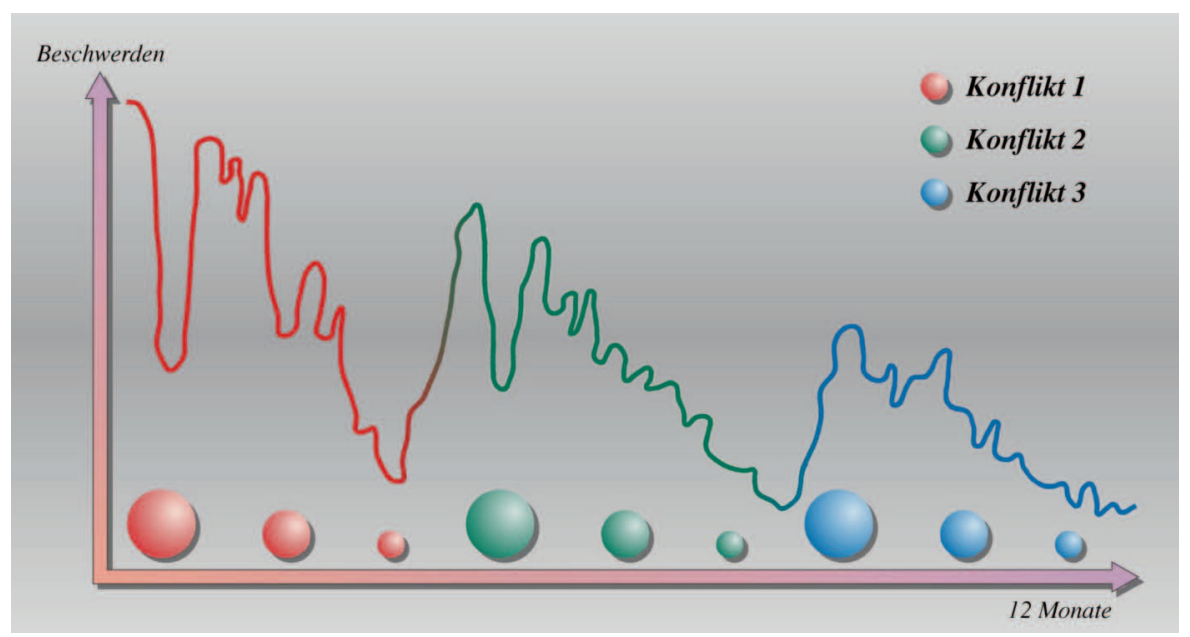

aber normalerweise weitgehend unbemerkt und unterschwellig.

In der Regel müssen zwei bis drei Konflikte aufgelöst werden, um einen dauerhaften und guten Erfolg zu erzielen (Abb. 3). Bei fast allen Patienten testet man anfangs nur einen einzigen Konflikt, ganz selten zwei. Das Auflösen des ersten Konfliktes dauert meist 4-5 Monate. Anschliessend meldet sich oft ein neues Konfliktthema, das wieder mehrere Monate der Therapie benötigt. Der ganze Vorgang ähnelt dem Pellen von seelischen «Zwiebelschalen». Es ist ein psychoenergetischer Wachstumsprozess, der meist langsam und unmerklich - selten dramatisch - zu einer Verbesserung des Gesamtzustands führt. Bei Kindern und seelisch sehr offenen Menschen kann der Heilungsprozess deutlich schneller gehen und endet gelegentlich bereits nach Auflösen eines einzigen Konflikts. Auch bei Tieren beobachten wir immer wieder erstaunlich schnelle Genesungen.

\section{Zertifizierte Energietherapeuten}

Das System der PSE wurde 1997 erstmals der medizinischen Öffentlichkeit vorgestellt. Es hat sich in kurzer Zeit zu einem Standardverfahren der Alternativmedizin entwickelt. Besonders verbreitet ist die PSE im deutsch- sprachigen Raum und zunehmend auch in Nordamerika. Über 2000 Therapeuten in 20 Ländern arbeiten mit dieser Methode. Aktuell sind ca. 500 «Energietherapeuten» speziell in der Methode ausgebildet und zertifiziert worden. Sie haben eine intensive theoretische und praktische Schulung absolviert und ihr Wissen in der anschliessenden Prüfung unter Beweis gestellt. Jeder zertifizierte Energietherapeut hat selbst eine Behandlung mit PSE durchlaufen, um eigene Energieblockaden abzubauen. Die Erfahrung hat gezeigt, dass nur dadurch zuverlässige Testergebnisse erzielt werden. Ein intensives Praxistraining rundet die mehrjährige Ausbildung ab.

Immer mehr Therapeuten entdecken, dass man mit PSE nicht nur seelische Störungen behandeln kann, sondern viele Menschen vom Auflösen der Konflikte und vom Wiederherstellen des energetischen Gleichgewichts profitieren können. Selbst bei hartnäckigen somatischen Erkrankungen wie Ulcus cruris oder Psoriasis erlebt man immer wieder gute Erfolge. Es handelt sich daher bei der PSE um eine ganzheitliche Behandlung. Darüber hinaus kann sie zur typgerechten Lebensberatung und als Grundlage zur Persönlichkeitsentwicklung dienen.
Abb. 3. Typischer Heilverlauf der PSE.

\section{Psychosomatische Energetik als Basistherapie}

Grundsätzlich kann die PSE mit praktisch allen gängigen Therapien kombiniert werden. Besonders Psychotherapeuten heben hervor, dass Patienten mithilfe der PSE schneller «auf den Punkt kommen». Das konsequente Auflösen der Konflikte erweist sich als eine ursächliche naturheilkundliche Therapie, weil die Heilungsprozesse zielgerichtet wirken und meist von dauerhafter Wirkung sind. Die seelischen Selbstheilungskräfte werden durch die PSE angeregt und Menschen werden motiviert, ihr Leben wieder selbst in die Hand zu nehmen. Häufig erlebt man, dass keine weiteren Therapiemethoden nötig sind, um eine vollständige Genesung zu erreichen, wobei sich die PSE grundsätzlich mit sehr vielen Heilverfahren sinnvoll kombinieren lässt. Bei der Mehrzahl der Patienten kann die PSE dabei als Basismethode eingesetzt werden.

\section{Literatur}

Banis R: Durch Energieheilung zu neuem Leben, ed 3. Petersberg, Via Nova, 2002.

Banis R: Psychosomatische Energetik - Lehrbuch für Therapeuten, ed 2. Kirchzarten, VAK, 2003.

Banis U: Wie wirkt Psychosomatische Energetik? Alles Wissenswerte zum Energie-Check und zur Therapie. Kirchzarten, VAK, 2010. 